\title{
Endoscopic airway equipment part 1: physical principles
}

\author{
R Hofmeyr, (iD A Elhouni iD \\ Department of Anaesthesia and Perioperative Medicine, Faculty of Health Sciences, University of Cape Town, and Groote Schuur Hospital, \\ Cape Town, South Africa \\ Corresponding author, email: ross.hofmeyr@uct.ac.za
}

\section{Summary}

The use of advanced endoscopic airway equipment has become increasingly important to the provision of safe anaesthesia for patients with complex anatomical and pathological conditions. Fundamental to the correct selection and use of the equipment is an understanding of the physical properties underlying its construction and function. This relies primarily on conventional optics, fibreoptics, video sensors and light-emitting diode technology.

Keywords: airway management, anaesthesia equipment, endoscopy, intubation, bronchoscopy

This review is intended to be read in conjunction with the accompanying "Endoscopic airway equipment part 2: equipment overview." An earlier unreferenced version was published as course notes accompanying the 2017 FCA 1 Refresher Course workshop of the same title.

\section{Introduction}

Safe, reliable airway management is integral to the practice of anaesthesia. ' While some procedures may be accomplished without advanced instrumentation of the airway, there are many in which the ability to accurately position endotracheal and endobronchial tubes, bronchial blockers, dilators and other devices is crucial to success, and ultimately, patient outcomes. ${ }^{2}$ The steady growth and refinement of endoscopes used to see within the airway has developed from simple tubes and reflected light-sources to sophisticated hybrid devices using fibre-optics and integrated video camera systems. A basic understanding of the physical properties that underlie the construction and function of these devices is essential to proper use and care. This review aims to give a brief introduction to the important physical principles, and is accompanied by a second part which gives a broad overview of the main types of endoscopic airway equipment in use. More information, training materials and video tutorials can be found on the open-access airway education web site, www.openairway.org. Specific queries can be directed to the authors.

\section{Basic principles of conventional optics}

Optics is that branch of physics which describes the characteristics and behaviour of light, instruments that use, detect or manipulate light, and its interactions with other forms of matter. Conventionally, it refers to visible, infrared and ultraviolet light, but as light is a form of electromagnetic wave, it has implications for other forms, such as x-rays, radio and microwaves.

Conventional geometrical optics describe the phenomena that can be accounted for using the classical electromagnetic ray form of light, which presumes travel in straight lines (or "rays"), and reflection or refraction when meeting or passing through
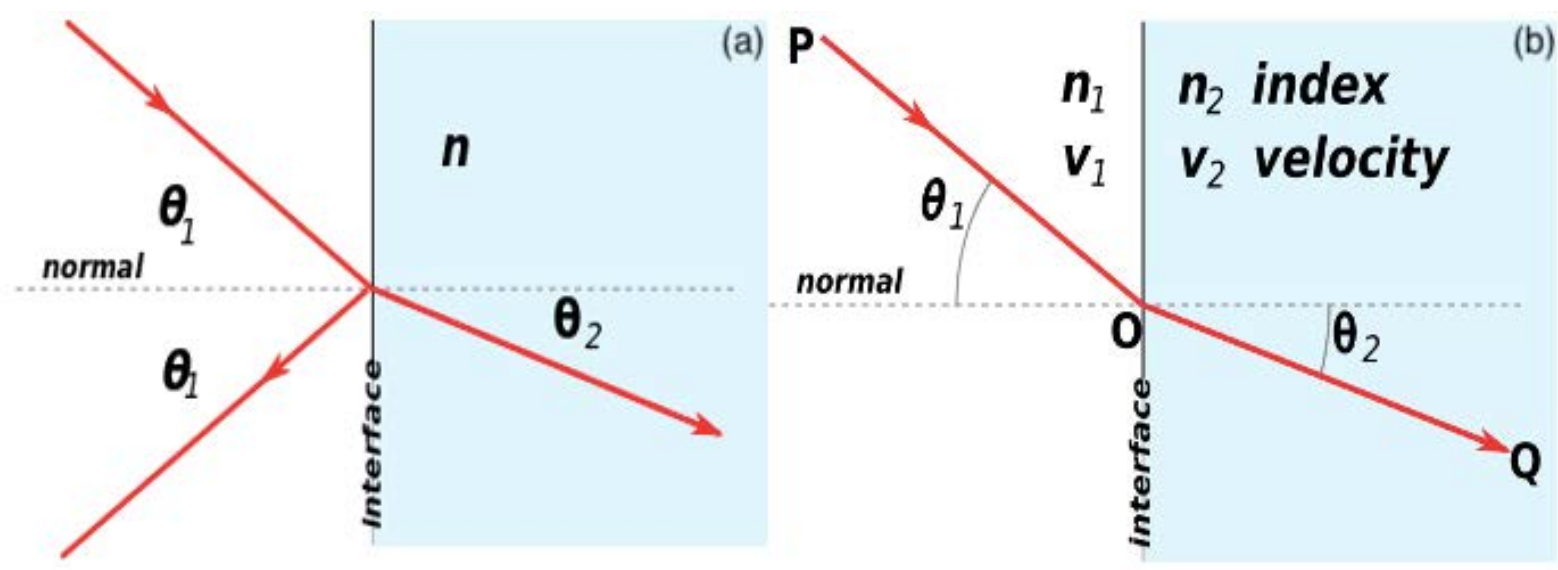

Figure 1: (a) Geometry of reflection and refraction (b) Snell's law of refraction 
surfaces. ${ }^{3}$ Wave and particle effects such as interference and diffraction are described by the more comprehensive physical and quantum optics, but are largely beyond the scope of the application to airway endoscopes.

The fundamental principles of geometric optics are the laws of reflection and refraction. Angles of incidence, reflection and refraction are always measured from the normal, which is perfectly perpendicular to the interface. When a ray of light meets the interface between two transparent materials, it is split into reflected and refracted rays, so that:

The reflected ray lies in the plane of incidence, and the angle of reflection equals the angle of incidence. (Law of reflection)

The refracted ray lies in the plane of incidence, and the sine of the angle of refraction divided by the sine of the angle of incidence is a constant for any two materials and a given wavelength of light. (Law of refraction)

This can also be expressed as Snell's law, which describes the angles from normal for a light ray traversing from a medium with refractive index of $n_{1}$ to a medium with index $n_{2}$ :

$$
n_{1} \sin \theta_{1}=n_{2} \sin \theta_{2}
$$

The velocity $(v)$ of light in a transparent medium is determined by the nature of the medium, but is always less than the speed of light through a vacuum. The refractive index $n$ for any given medium is therefore calculated as

$$
\boldsymbol{n}=\boldsymbol{c} / \boldsymbol{v}
$$

where $c$ is the speed of light through a vacuum. Where there is a marked difference between the indexes of refraction from one medium to another, such as between glass and air, Snell's law predicts that there is no $\theta_{2}$ when $\theta_{1}$ is sufficiently large. In other words, when the angle of incidence is sufficiently far from the normal, no light is refracted, and total internal reflection occurs. This is the fundamental principle of the function of fibreoptics.

Lenses are devices which cause light rays to converge or diverge through refraction. Converging lenses focus incoming parallel rays onto a spot at focal length from the lens, on the opposite side. Diverging lenses spread the incoming parallel rays in such

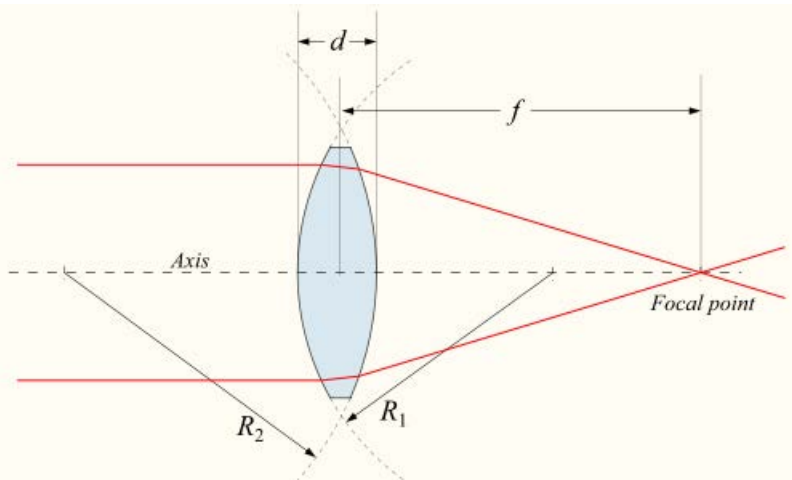

Positive (converging) lens

Figure 2: Converging lens (Image: Wikipedia/DrBob, CC-BY-SA 3.0) a way that they appear to have originated at a position one focal length on the same side as the origin.

Several types of indirect laryngoscope (whereby an image of the vocal cords is produced without a direct visual axis) make use of a combination of lenses, mirrors and/or prisms. Examples include the TruView ${ }^{\circledR}$ and Airtraq ${ }^{\circledR}$ optical laryngoscopes. ${ }^{4-8}$

\section{Principles of fibreoptics}

Optical fibres are flexible structures created by sequentially drawing transparent glass or plastic into a very fine diameter filament, often comparable to (or thinner than) a single human hair. Using the principle of total internal reflection, they allow transmission of light from one end to the other, potentially over long distances, with minimal loss of intensity. This is referred to as acting as a waveguide, and allows transmission of light (and therefore, images) around corners. ${ }^{9}$ Often, cladding material with a lower index of refraction is used around the optical fibre to further increase efficiency. They have wide-ranging uses in illumination, imaging, sensing and communications. Increasingly, optical fibres are used in the installation of fixed communications systems within healthcare and other facilities, and for long-distance data transmission, due to greater bandwidth with much less signal loss.

In medical endoscopes, optical fibre bundles (frequently consisting of tens of thousands of individual fibres) are used both to provide illumination and to produce an image. Non-coherent fibre bundles convey light from a light source distant from the patient to the end of the scope, thereby avoiding having a bulb at the scope's tip, which would produce heat and could cause burns. To convey an image, coherent bundles (where the fibres maintain their same perfect position parallel to each other from one end of the scope to the other) are used. An objective lens at the tip of the endoscope is used to focus the image on the fibre bundle, and an eyepiece at the user's end allows viewing the image.

Optical fibres allow for the creation of flexible endoscopes, and are used in some types of rigid endoscope for either light

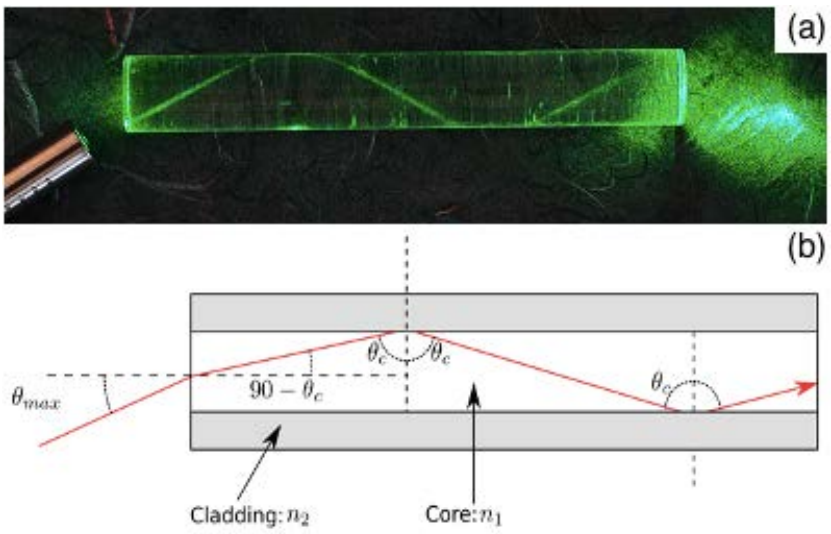

Figure 3: (a) A laser passing down a perspex rod illustrating the principle of total internal reflection of light in an optical fibre. (Wikipedia/Timwether, CC-BY-SA 3.0) (b) Schematic diagram of an optical fibre, showing the numerical aperture. Light undergoes total internal reflection due to Snell's law. (Wikipedia/User A1, CC-BY-SA 3.0) 


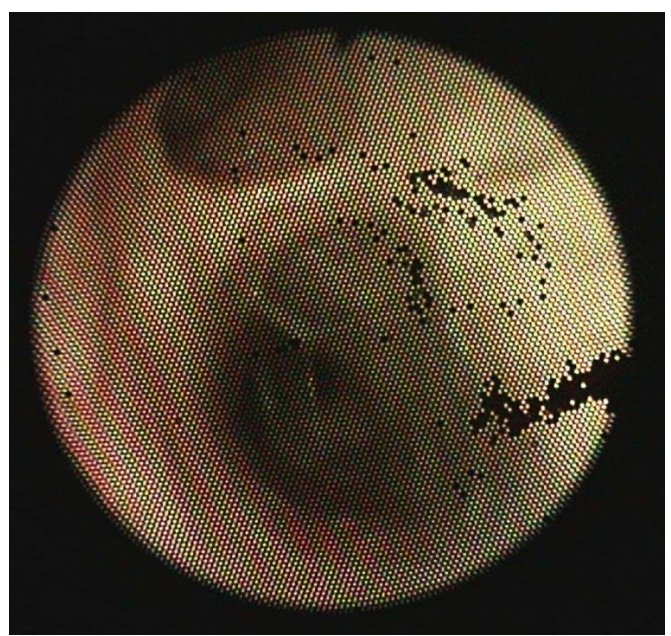

Figure 4: Fibreoptic image, showing typical honeycomb appearance and several 'dead' fibres.

conduction, or both light and image conduction. A fibreoptic image is easily identifiable by the 'pixelated' appearance, as each individual 'pixel' is an individual fibre. Due to the very fine nature of optical fibres, however, they are susceptible to breaking through tight bends, hard knocks and crushing. Individual broken fibres can be seen in the image as black 'dead' spots. As the number of broken fibres increases, the scope eventually becomes unusable. (Figure 4)

\section{Video camera and display integration into endoscopes}

The value of performing endoscopy is greatly magnified if the image can be enlarged, displayed on a screen to enable multiple simultaneous viewers, and recorded for documentation, teaching and medicolegal purposes. The initial method to achieve this was to mount a still or video camera onto the eyepiece of an optical scope, and link the camera to a display and/or video recorder. Using modern rod lens telescopes in combination with a highdefinition (HD) camera, very high-quality images can be recorded and displayed. One downside to this system, however, is that the weight of the camera can make holding and manipulating the endoscope more difficult and tiring.

Using an HD camera with a flexible fibreoptic scope is less effective, as the resolution is limited by the density of the fibre bundles themselves. This is often visible as a prominent 'honeycomb' pattern on the screen, which can make viewing difficult. To counter the honeycomb effect, slightly defocussing the scope, or the use of advanced imaging filters which interpolate between pixels, can be used.

An alternate approach is to completely replace the optical fibres in flexible endoscopes with a digital video system. The advent of very small video camera sensors - and rapid improvement in their quality - combined with a dramatic reduction in cost has largely been fuelled by the digital camera and mobile phone industries. This has made these 'chip-in-tip' endoscopes increasingly common. The light source and non-coherent fibre bundles are also replaced by one or more light-emitting diodes (LEDs) at the end of the scope, which makes flexible video endoscopes lighter,

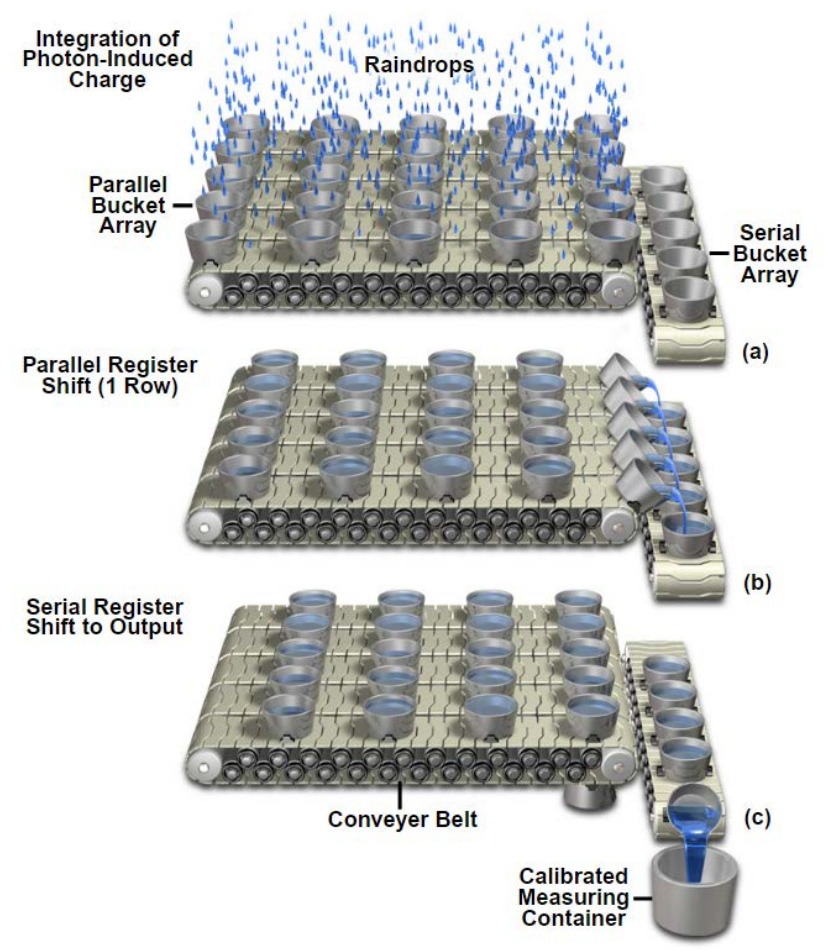

Figure 5: CCD 'Bucket Brigade' analogy: Individual pixels (buckets) collect incoming photons (raindrops) in the form of electrical charge. This is then transferred across the array in orderly steps and recorded sequentially into a digital signal.

Image courtesy of https://www.microscopyu.com

more robust, and thinner in diameter. Alternately, the same diameter as a fibreoptic scope can be achieved with a larger working channel.

The original video chips were of the charge-coupled device (CCD) design. A thin silicon wafer is divided into a geometric array of light-sensitive regions (picture elements, or 'pixels') that locally store a charge dependent on the degree of light exposure. After exposure, the charge accumulated by each pixel is transferred across the array in sequence, creating a digital signal which encodes the image. This has been likened to an array of buckets collecting rain. (Figure 5)

A second type of digital image chip is the complementary metaloxide semiconductor (CMOS) sensor, which employs a similar array architecture but allows multi-channel recording from the matrix, greatly increasing image capture speed. CCD sensors are more sensitive to light than CMOS, and therefore produce images with less 'noise' at the same light intensity. However, in the lightabundant environment of airway endoscopy, this is much less of a disadvantage. CMOSs, however, are more power-efficient and can be produced at much lower cost. Steady improvements in CMOS sensor quality has led to their domination of the camera chip market, and most modern video endoscopes use a CMOS chip.

Light-emitting diodes (LEDs) are electroluminescent light sources, in which the interface between two semiconductor materials produces photons when subjected to a suitable voltage. They have multiple advantages for the use in 


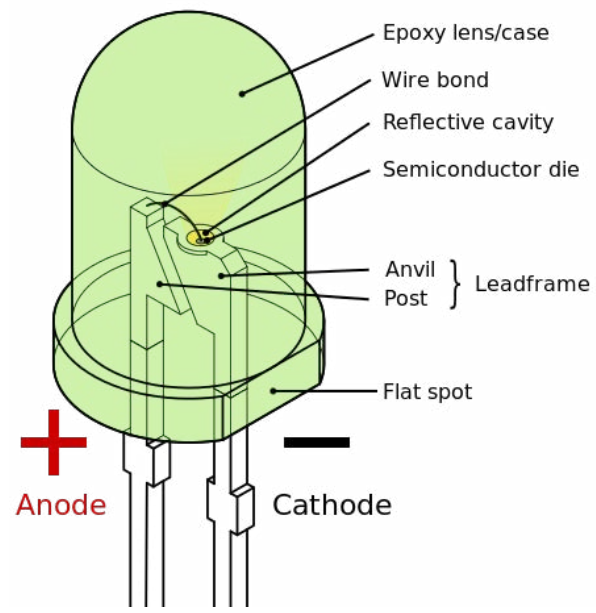

Figure 3: Schematic of a light-emitting diode

endoscopes of all types when compared to incandescent and fibreoptic light sources: small size (less than $1 \mathrm{~mm}^{2}$ ), low power consumption, long lifetime (often measured in tens of thousands of hours), physical robustness, and little or no heat production. Although the advent of fibreoptic light guides ushered in the concept of 'cold light sources', only the incorporation of LEDs into endoscopes has truly achieved this goal.

Continual improvement in quality and reduction in both cost and size of both image sensor chips and LEDs has led to an explosion in their use in both flexible endoscopes and video laryngoscopes.

\section{Conclusion}

The steady development and refinement in airway endoscopes - including the steady progression from reliance on traditional fibreoptic and conventional optics devices towards increasingly capable video devices - will with time expand the capabilities of practitioners and reduce the costs/barriers to access. Future advances may allow integration with artificial intelligence, machine learning, data assimilation and improved patient safety. Anaesthesiologists who wish to master the airway must be familiar with the basic principles, strengths and weaknesses of the equipment. It behoves us to recall that, more important than the tool itself, is the one that holds the tool.

\section{Conflict of interest}

$\mathrm{RH}$ directs the UCT-Storz African Fellowship in Airway and Thoracic Anaesthesia, which is supported in part by an unrestricted educational grant to the University of Cape Town by Karl Storz GmBH. AE is the incumbent Fellow at the time of writing. No commercial entity had any role in the production or publication of this manuscript. Inclusion of images or mention of specific products by name in this article is for the purposes of discussion and is not an endorsement of the product.

\section{ORCID}

R Hofmeyr (iD https://orcid.org/0000-0002-9990-7459

A Elhouni (iD https://orcid.org/0000-0003-4970-6592

\section{References}

1. Frerk C, Mitchell VS, McNarry AF, et al. Difficult Airway Society 2015 guidelines for management of unanticipated difficult intubation in adults. Br J Anaesth. 2015;115(6):827-48. https://doi.org/10.1093/bja/aev371.

2. Ahmad I, El-Boghdadly $K$, Bhagrath $R$, et al. Difficult Airway Society guidelines for awake tracheal intubation (ATI) in adults. Anaesthesia. 2020;75(4):509-28. https:// doi.org/10.1111/anae.14904.

3. Wikipedia. Geometrical Optics. 14 July 2020. Available from: https:// en.wikipedia.org/wiki/Geometrical_optics. Accessed 30 September 2020.

4. Nasim S, Maharaj $\mathrm{CH}_{\text {, Butt I, et al. Comparison of the Airtraq }}{ }^{\circledR}$ and Truview ${ }^{\circledR}$ laryngoscopes to the Macintosh laryngoscope for use by Advanced Paramedics in easy and simulated difficult intubation in manikins. BMC Emerg Med. 2009;9:2. https://doi.org/10.1186/1471-227X-9-2.

5. Maharaj $\mathrm{CH}, \mathrm{O}^{\prime}$ Croinin D, Curley G, Harte BH, Laffey JG. A comparison of tracheal intubation using the Airtraq or the Macintosh laryngoscope in routine airway management: a randomised, controlled clinical trial. Anaesthesia. 2006;61(11):1093-9. https://doi.org/10.1111/j.1365-2044.2006.04819.x.

6. Maharaj $\mathrm{CH}$, Buckley E, Harte BH, Laffey JG. Endotracheal intubation in patients with cervical spine immobilization: a comparison of Macintosh and Airtraq laryngoscopes. Anesthesiology. 2007;107(1):53-9. https://doi.org/10.1097/01. anes.0000267529.71756.f0.

7. Lu Y, Jiang $\mathrm{H}$, Zhu YS. Airtraq laryngoscope versus conventional Macintosh laryngoscope: a systematic review and meta-analysis. Anaesthesia. 2011;66(12):1160-7. https://doi.org/10.1111/j.1365-2044.2011.06871.x.

8. Hoshijima H, Mihara T, Denawa $\mathrm{Y}$, et al. Airtraq ${ }^{\circledR}$ is superior to the Macintosh laryngoscope for tracheal intubation: systematic review with trial sequential analysis. Am J Emerg Med. 2019;37(7):1367-8. https://doi.org/10.1016/j. ajem.2018.12.018.

9. Davis P. Basic physics and measurement in anaesthesia. 5th ed. ButterworthHeinemann; 2003. 